\title{
IRES UNPLUGGED
}

Fátima Gebauer ${ }^{1,2}$ and Matthias W Hentze ${ }^{3}$

(1) Gene Regulation, Stem Cells and Cancer Programme, Centre for Genomic

Regulation (CRG), The Barcelona Institute of Science and Technology, 08003Barcelona, Spain

(2) Universitat Pompeu Fabra (UPF), 08003-Barcelona, Spain

(3) European Molecular Biology Laboratory (EMBL), Meyerhofstrasse 1, 69117-

Heidelberg, Germany 
All cellular mRNAs contain a m7GpppN cap structure at their $5^{\prime}$ ends which promotes efficient translation and mRNA stability. The cap is recognized by the translation initiation factor eIF4F that attracts the 40S small ribosomal subunit, which then scans the 5' UTR towards the initiation codon for subsequent protein synthesis. In conditions of stress or during viral infections, cap-dependent translation is compromised. Internal ribosome entry sites (IRESs) can bypass the cap to recruit ribosomes internally to the message helping to secure appropriate protein synthesis. In this issue of Science, Segal and colleagues report the systematic discovery of cellular and viral IRESs, identifying a significant fraction of mRNAs $(\sim 10 \%)$ and viruses $(\sim 20 \%)$ with the potential to be translated by this cap-independent mechanism (1).

IRESs were first discovered as complex structures in the 5' UTRs of Picornavirus transcripts, and were later found to occur in other viral and in cellular mRNAs (2). IRESs interact with translation initiation factors, with bridging RNA-binding proteins or with the small ribosomal subunit itself, leading to ribosomal positioning at or near the initiation codon, and typically promoting translation initiation with little or no ribosomal scanning. IRESs have also been found as short sequence elements that can base pair with rRNA similar to Shine-Dalgarno sequences in bacterial translation initiation (3). IRESs lack common features that allow bioinformatic prediction, calling for experimental approaches.

The standard test to detect IRES activity within a given sequence is the bicistronic reporter assay, where the candidate sequence is placed between two cistrons and is tested for its ability to direct significant translation of the downstream cistron. This assay must be accompanied by rigorous controls to exclude alternative explanations (4), especially the possibility that monocistronic transcripts encoding the second cistron are produced from cryptic promoters or by splicing within the tested sequence. The typically modest activity of cellular IRESs in the bicistronic assay or the omission of controls have fueled skepticism regarding the prevalence of IRESs in cellular mRNAs. However, the existence of ribosomal proteins seemingly dedicated to IRES-dependent translation support IRESs as relevant effectors of cellular mRNA translation $(5,6)$. Segal and colleagues established a high-throughput bicistronic reporter assay to quantify IRES-dependent translation driven by thousands of fragments of $174 \mathrm{nt}$ in length derived from cellular and viral mRNAs. A set of 583 cellular and 471 viral novel 5' UTR IRESs emerged, passing controls for cryptic promoter or splice site artifacts.

As one of the most intriguing aspects of the study, mutagenesis experiments yielded an IRES classification into two types: "global" IRESs where complex and extended RNA structures are required to drive cap-independent translation, and "local" IRESs defined by - often U-rich - short sequence motifs typically located within $60 \mathrm{nts}$ upstream of the initiation codon. Interestingly, some of these sequences display complementarity to a region of 18S rRNA, helices h23 and h26, which was previously shown to contact mRNA (Figure 1). Using scanning oligonucleotides with complementarity to the entire 18S rRNA sequence in the bicistronic assay, a distinct region of 18S rRNA (nts 812-1233) including h23 and 
h26 was systematically defined as "active" for mediating IRES function by mRNA complementarity. While the "global" IRESs suggest that the interaction with translation initiation or other bridging RNA-binding factors aid in ribosome recruitment, the "local" IRESs could in large part be based on Shine-Dalgarnolike mRNA-rRNA complementarity.

IRESs populate not only the 5' UTR but also the ORF itself and, surprisingly, the 3' UTR. While the latter is intriguing and may suggest "shunting" of ribosomes from the 3'UTR to the initiation codon, possibly mediated by mRNA circularization (7), their unnatural placement upstream of an ORF in the bicistronic reporter assay calls for follow-up work that evaluates these sequences within their natural contexts. Furthermore, the 3' UTR may contain cap-independent translation enhancers (CITEs), which can also function when placed at the 5' UTR (8). CITEs, found in plant viruses, recruit translation initiation factors and deliver them to the $5^{\prime}$ end of the mRNA via long distance base-pairing with the 5' UTR. Translation from CITEs requires scanning and, thus, are different from IRESs. Although they generally do not score in the bicistronic reporter assay, they might do so in combination with other sequences.

The report by Segal and colleagues suggests that IRES-mediated translation is also widespread amongst viral RNAs. Fragments with IRES activity have been found along the polyprotein region of several viruses, implying that IRESs might drive the independent expression of viral proteins from within the major polyprotein ORF. This finding may stimulate research into new anti-viral strategies.

Recent findings indicate that adenosine methylation $\left(\mathrm{m}^{6} \mathrm{~A}\right)$ in the $5^{\prime}$ UTR can also stimulate cap-independent translation through interactions with eIF3 (9). This type of translation differs from IRES-driven translation, because it requires scanning and a free $5^{\prime}$ end. Together with (1), these recent reports illustrate the diversity of cap-independent translation initiation mechanisms in mammalian cells. As we begin to gain detailed molecular understanding of the standard capdependent translation initiation process, these developments highlight that much remains to be discovered mechanistically and about the biology of alternative forms of translation.

\section{References}

1. S. Weingarten-Gabbay et al., Science (2015)

2. R. J. Jackson,. Biochem Soc Trans. 33, 1231-1241 (2005)

3. J. Dresios, S. A. Chappell, W. Zhou, V. P. Mauro, Nat. Struct. Mol. Biol. 13, 30-34 (2006)

4. S. R. Thompson, Wiley Interdiscip Rev RNA. 3, 697-705 (2012)

5. D. M. Landry, M. I. Hertz, S. R. Thompson, Genes Dev. 23, 2753-2764 (2009)

6. S. Xue et al., Nature 517, 33-38 (2015)

7. S. E. Wells, P. E. Hillner, R. D. Valle, A. B. Sachs, Mol Cell 2, 135-140 (1998)

8. I. N. Shatsky, S. E. Dmitriev, D. E. Andreev, I. M. Terenin, Crit Rev Biochem Mol Biol. 49, 164-177 (2014) 
9. K. D. Meyer et al., Cell 163, 999-1010 (2015)

\section{Figure caption}

IRESs can direct cap-independent translation from various locations in the mRNA. Two types of IRESs are depicted, "global" (green) and (U-rich) "local" IRESs (pink), frequently with complementarity to the "active region" (yellow) of $18 S$ rRNA. 


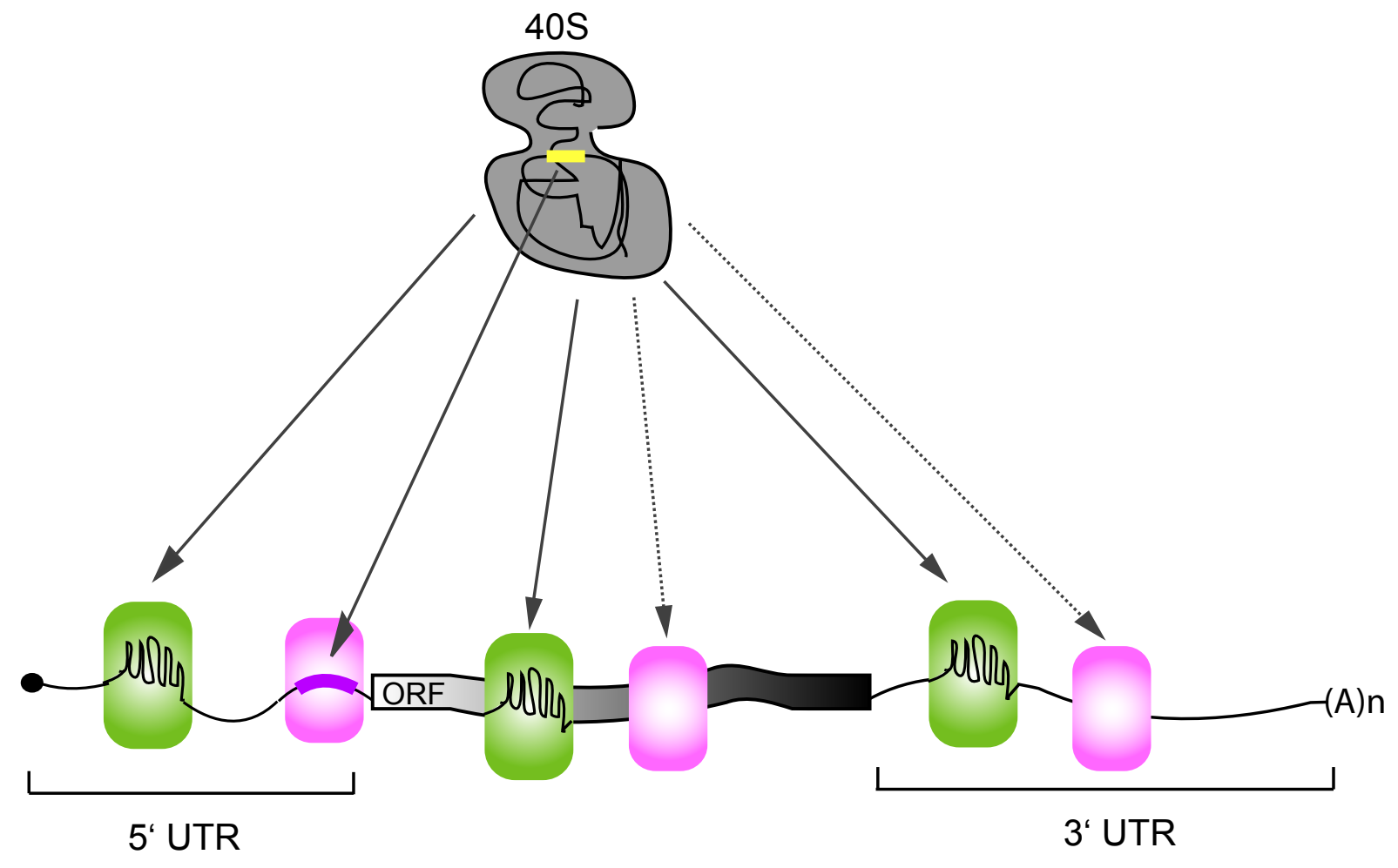

